University of Wollongong

Research Online

Faculty of Engineering and Information

Faculty of Engineering and Information

Sciences - Papers: Part A

Sciences

$1-1-2012$

\title{
The use of transient state estimation for voltage dip/sag assessment
}

\author{
A Farzanehrafat \\ University of Canterbury \\ N R. Watson \\ University of Canterbury \\ S Perera \\ University of Wollongong, sarath@uow.edu.au
}

Follow this and additional works at: https://ro.uow.edu.au/eispapers

Part of the Engineering Commons, and the Science and Technology Studies Commons

Research Online is the open access institutional repository for the University of Wollongong. For further information contact the UOW Library: research-pubs@uow.edu.au 


\title{
The use of transient state estimation for voltage dip/sag assessment
}

\author{
Abstract \\ Voltage sags are common events on the electric power network caused by network faults and the large \\ load connections. This power quality issue can affect a wide range of electrical equipment malfunctions, \\ interruptions or losses and are of particular concern to industry. A complete knowledge of the source is a \\ prerequisite before any remedial action is taken. On the other hand, it is not feasible to fully monitor \\ voltage sags occurred throughout the network. For this reason state estimation techniques have been \\ extended into power quality issues and called Power Quality State Estimation (PQSE). This paper, \\ introduces the use of Transient State Estimation (TSE) as one type of PQSE for voltage dip/sag \\ assessment. The presented approach makes use of mismatch nodal voltage to identify the type and \\ location of voltage dip/sag origin via limited number of measurement points within the network. Then, \\ this algorithm is applied to a distribution test system to verify its performance in presence of \\ measurement noise. (c) 2012 IEEE.
}

\section{Keywords}

sag, assessment, state, dip, transient, voltage, estimation

Disciplines

Engineering | Science and Technology Studies

\section{Publication Details}

A. Farzanehrafat, N. R. Watson \& S. Perera, "The use of transient state estimation for voltage dip/sag assessment," in 2012 IEEE International Conference on Power System Technology, POWERCON 2012, 2012, pp. 1-6. 


\title{
The Use of Transient State Estimation for Voltage Dip/Sag Assessment
}

\author{
A. Farzanehrafat, Student Member, N. R. Watson, Senior Member, S. Perera, Member, IEEE
}

\begin{abstract}
Voltage sags are common events on the electric power network caused by network faults and the large load connections. This power quality issue can affect a wide range of electrical equipment malfunctions, interruptions or losses and are of particular concern to industry. A complete knowledge of the source is a prerequisite before any remedial action is taken. On the other hand, it is not feasible to fully monitor voltage sags occurred throughout the network. For this reason state estimation techniques have been extended into power quality issues and called Power Quality State Estimation (PQSE). This paper, introduces the use of Transient State Estimation (TSE) as one type of PQSE for voltage dip/sag assessment. The presented approach makes use of mismatch nodal voltage to identify the type and location of voltage dip/sag origin via limited number of measurement points within the network. Then, this algorithm is applied to a distribution test system to verify its performance in presence of measurement noise.
\end{abstract}

Index Terms-- Voltage Dip/Sag origin, Transient State Estimation, Power Quality State Estimation.

\section{INTRODUCTION}

$\mathrm{V}$ OLTAGE Dip/Sag is one of the most concerned power quality issues and as defined by [1], is a sudden decrease in RMS voltage at the power frequency for durations from 0.5 cycles to 1 minute, reported as the remaining voltage. With the growing number of hi-tech but sensitive devices, this reduction may result in an unexpected stoppage when such sensitive equipment is located in a process-controlled device within an industrial plant. As mitigation plans must be carried out, locating the source of disturbance has to be done as a first step. For these reasons, many techniques have further been developed to identify the source of voltage dip/sag in power systems.

Stochastic prediction techniques [2-10] combine mathematical models and statistical data to predict the number and characteristics of voltage dips experienced by a customer

Ali Farzanehrafat is a Ph.D. candidate at the Electrical \& Computer Engineering Department, University of Canterbury, Christchurch 8020, New Zealand and a visiting research student at University of Wollongong, NSW 2500, Australia (e-mail: ali.farzanehrafat@pg.canterbury.ac.nz).

Neville R. Watson is a Professor at the Electrical \& Computer Engineering Department, University of Canterbury, Christchurch 8020, New Zealand (e-mail: neville.watson@canterbury.ac.nz).

Sarath Perera is an Associate Professor at the School of Electrical, Computer and Telecommunication Engineering, University of Wollongong, NSW 2500, Australia (e-mail: sarath@uow.edu.au).

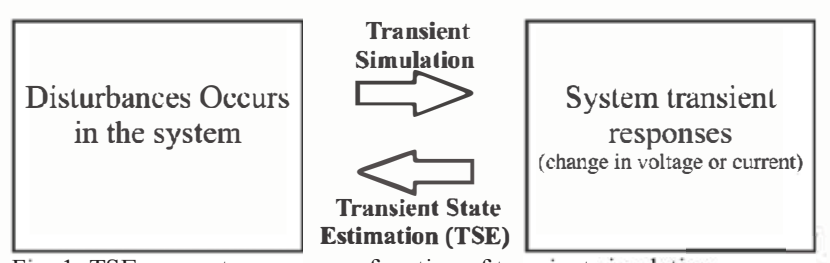

Fig. 1: TSE concept as a reverse function of transient simulation.

fed from a certain feeder in a network. However, due to the fact that assessment of voltage dips/sags requires prior knowledge of the system and is based on stochastic evaluation, the problem of locating voltage sag origin remains uncertain.

Many contributions also used different methods to identify the location of voltage sags origin [11-19]. Apart from advantages and disadvantages associated with each method, the common feature of these methods are to determine the disturbance origin is located upstream or downstream regarding the measuring point. This approach renders useful information particularly for industrial customers who are interested to identify responsible party for the voltage sag occurred within their plant. However, it still remains at a disadvantage from the network operator point of view.

Recently, the importance of power quality issues and the reduction in price of meters capable of measuring power quality indices has resulted in research being focused on extending the concept of state estimation techniques into power quality issues. This area of research is called Power Quality State Estimation (PQSE) [20]. This paper, introduces the use of Transient State Estimation (TSE) [21-22]as one type of PQSE for voltage dip/sag assessment. TSE provides a platform in which particular information of interest about power quality issues including voltage dips/sags can be easily extracted via limited number of measurement points throughout the network.

In essence, TSE is a reverse function of transient simulation (Fig. 1). While transient simulation is used to analyse the consequences of a disturbance on a power system voltage, current, etc., TSE is exploited to identify the cause of transient change in system parameters. Therefore, TSE can be used as a valuable tool to identify type and location of the disturbance in a power system.

Section II, describes the methodology for TSE with a brief explanation about how TSE is formulated by means of NIS. SVD as a method of choice is discussed and the observability criteria are addressed. Then, in section III, the implemented algorithm is applied to a distribution system to verify its 
performance. Its ability to estimate busbar voltages and thereby location of the source of a voltage dip/sag using nodal mismatch voltage is used as the test. Concluding comments are given in section IV.

\section{MEthodology}

Transient variations in TSE necessitate a time-domain solution for the system as well as a dynamic formulation to represent system components. Numerical Integrator Substitution (NIS), also known as Dommel's method [23], has been employed for modelling the system components in time domain for TSE in order to develop the measurement equation.

Dommel's EMTP approach combines the method of characteristics for transmission lines and trapezoidal rule to convert differential equations of system components into algebraic equations involving voltages, currents and past values. This results in a Norton equivalent shown in Fig. 2 for each system component. The conductance $\left(G_{\text {eff }}\right)$ represents instantaneous term relating the current contribution to the voltage at the present time step. The current source $\left(I_{\text {History }}\right)$ represents history term which is the contribution to current from the previous time step values:

For a resistance:

$$
i_{k m}(t)=G_{e f f}\left(v_{k}(t)-v_{m}(t)\right)
$$

For a Capacitor:

$$
\begin{aligned}
& i_{k m}(t)=G_{\text {eff }}\left(v_{k}(t)-v_{m}(t)\right)+I_{\text {History }} \\
& I_{\text {History }}=-G_{\text {eff }}\left(v_{k}(t-\Delta t)-v_{m}(t-\Delta t)\right)-i_{k m}(t-\Delta t)
\end{aligned}
$$

For an Inductor:

$$
\begin{aligned}
& i_{k m}(t)=G_{\text {eff }}\left(v_{k}(t)-v_{m}(t)\right)+I_{\text {History }} \\
& I_{\text {History }}=i_{k m}(t-\Delta t)+G_{\text {eff }}\left(v_{k}(t-\Delta t)-v_{m}(t-\Delta t)\right)
\end{aligned}
$$

The power system can be described as a set of interconnected RLC branches. So, time-domain solution for each system component can be written individually using equations (1) - (3). Considering phase to ground voltages (hereafter called nodal voltages) as variables and based on the network topology, the nodal solution is applied to formulate the dynamic model of each system component as well as the entire network. It leads to a coherent nodal system of equations to be solved as follows:

$$
[G] \cdot \boldsymbol{v}(t)=\boldsymbol{i}_{\boldsymbol{s}}(t)-\boldsymbol{I}_{\text {History }}
$$

where $[G]$ is the conductance matrix, $v(t)$ is the vector of nodal voltages, $\boldsymbol{i}_{\boldsymbol{s}}(t)$ is the vector of external current sources and $\boldsymbol{I}_{\text {History }}$ is the vector current sources representing past history terms. Reference [24] provides comprehensive information on different aspects of system component modelling using this approach.

\section{A. Construction of TSE Problem:}

The general form of the state estimation problem can be expressed as equation (3). " $n$ " is assumed as the number of nodal voltages existing in the system we are looking for and " $\mathrm{m}$ " is assumed as the number of measurements taken from the system.

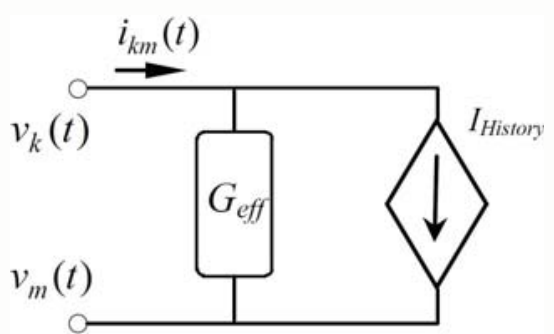

Fig. 2: Norton Equivalent circuit diagram, where the conductance $G_{\text {eff }}$ equals $I / R$ for a resistance, $2 C / \Delta t$ for a capacitor and $\Delta t / 2 L$ for an inductor.

$$
z=[H] \cdot \boldsymbol{x}+\boldsymbol{\varepsilon}
$$

where $\boldsymbol{z}$ is a $(\mathrm{m} \times 1)$ vector of measured quantities and $\boldsymbol{x}$ is a $(\mathrm{n} \times 1)$ vector of state variables (unknown quantities) for which the equation must be solved. $[H]$ is a $(m \times n)$ measurement function relating the known quantities to state variables and $\varepsilon$ is the vector of measurement errors. For TSE, nodal voltages and branch and load currents are measured quantities and state variables are nodal voltages.

To construct the measurements matrix, $[\mathrm{H}]$, each measurement point results in one equation that adds corresponding row from dynamic model into. For example, when a node voltage is measured the corresponding row in the [H] matrix will comprise of all zeros except at the position corresponding to the node that was measured (where the entry will be one) or when a branch current measurement will add a row to $[\mathrm{H}]$ consisting of all zeros except at the locations associated with the sending and receiving end nodes, which will contain the appropriate conductance entries from the [G] matrix.

To illustrates how relevant entries must be selected for the fundamental electrical circuit elements previously described and the relevant values defined in equations (1) - (3), following cases are considered for an arbitrary taken measurement number " $r$ ":

For instantaneous nodal voltage $\left(v_{k}\right)$ measurement:

$$
\begin{aligned}
& z_{r}(t)=v_{k}(t) \\
& H_{r, k}=1.0
\end{aligned}
$$

For instantaneous voltage measurement across $\mathrm{R}, \mathrm{L}$ or $\mathrm{C}$ between nodes $k$ and $m$ :

$$
\begin{aligned}
& z_{r}(t)=v_{k}(t)-v_{m}(t) \\
& H_{r, k}=1.0, H_{r, m}=-1.0
\end{aligned}
$$

For instantaneous current measurement through $\mathrm{R}, \mathrm{L}$ or $\mathrm{C}$ from sending node $m$ to receiving node $k$ :

$$
\begin{aligned}
& z_{r}(t)=i_{\text {measured }}(t)-I_{\text {History }} \\
& H_{r, k}=G_{e f f}, H_{m, k}=-G_{e f f}
\end{aligned}
$$

Basically, the system is over-determined, determined or under-determined depends on the number of measurements compared to the number of nodal voltages (rank of $[\mathrm{H}]$ ). Locating of voltage sag/dip origin leads to an underdetermined system of equations due to the cost of measurement devices capable of measuring PQ issues as well as different ownership of different parts of the system, thus observable and 
unobservable busbars are expected (partially observable system). In such cases, while most traditional estimation techniques fail, there is a robust approach to solve derived TSE problem.

\section{B. Solution of TSE:}

Once the state estimation problem is formed, it should be solved for state variables (nodal voltages). Among the suggested approach to solve the state estimation equation, using Singular Value Decomposition (SVD) is the selected approach in this paper.

1) Singular Value Decomposition (SVD): The SVD represents the measurement matrix, $[H](m \times n)$, as a product of three matrices as follows:

$$
[H]=[U] \cdot[W] \cdot[V]^{T}
$$

where $\mathrm{U}(m \times m)$ and $\mathrm{V}^{\mathrm{T}}(n \times n)$ are orthogonal matrices and $\mathrm{W}$ $(m \times n)$ is a diagonal matrix with entries of singular values of $\mathrm{H}$. This factored form can be calculated through eigenvalue analysis. $\mathrm{WW}^{\mathrm{T}}$ is an eigenvalue diagonal matrix. $[U]$ is the eigenvector matrix of $\mathrm{HH}^{\mathrm{T}}$ and its columns whose samenumbered elements of singular values are nonzero are an orthonormal set of basis vectors that span the range of matrix $\mathrm{H}$. [V] is the eigenvector matrix of $\mathrm{H}^{\mathrm{T}} \mathrm{H}$ and its columns whose same-numbered elements of singular values are zero are an orthonormal set of basis vectors that span the null space of matrix $H$. The inverse of measurement matrix and hence the solution can be calculated as follows:

$$
[H]^{-1}=[V] \cdot\left[\operatorname{diag}\left(1 / w_{j}\right)\right]^{-1} \cdot[U]^{T}
$$

If some of the $w_{j}$ 's $j=1,2, \ldots, n$ are zero or near zero, then measurement matrix is singular. In this case, a zero is placed in the diagonal element of $[\mathrm{W}]^{-1}$ (instead of $[1 / \mathrm{w}]$ ). Then, equation (5) with the singular $1 / w_{j}$ 's replaced with zero, called the pseudo-inverse of $[\mathrm{H}]$, are used to calculate state variables (nodal voltages):

$$
x=[V] \cdot\left[\operatorname{diag}\left(1 / w_{j}\right)\right]^{-1} \cdot[U]^{T} \cdot z
$$

Two scenarios are possible depends on whether or not $x$ relies in the range of $[\mathrm{H}]$. If it does, then the singular set of equations $(z=[H] . x)$ have more than one solution, since any vector in the null space can be added to $\mathrm{x}$ and gives another valid solution. However, (6) is able to produce the one shortest length among many. If $z$ is not in the range of $[\mathrm{H}]$, then the set of equations $(z=[H] . x)$ has no solution. In such a case, (6) can still be used to construct a "solution" vector $x$. This vector $x$, will not exactly solve estimation problem. But, among all possible vectors $\mathrm{x}$, it finds $x$ which minimizes $r \equiv|H \cdot x-z|$, where $r$ is called the residual of the solution [25].

2) Observability Criteria: Normal equation approach can be applied only when the system is fully observable and hence an inverse of matrix $\mathrm{H}$ exists. It requires prior Observability Analysis (OA) is performed to ensure the system is fully observable. However, SVD is able to give reliable answers for some state variables even when the system is partially observable. Moreover, inspection of the singular values and component matrices also gives important information on observability.

Basically, SVD is able to produce an infinite number of solutions that satisfies the equation, expressed by:

$$
[x]=\left[x_{p}\right]+\sum_{i=0}^{N} k_{i}\left[x_{n i}\right]
$$

where $\left[x_{p}\right]$ is the particular solution, $k_{i}$ is a constant and $\left[x_{n i}\right]$ is the null space vector. The number of null space vectors, $N$, is the number of zero $w_{j}$ 's in matrix $[W]$ equal to zero or equivalently ( $n$-rank $[H])$.

We already saw the columns of $[V]$ whose same-numbered elements of singular values are zero are an orthonormal set of basis vectors that span the null space of matrix $H$. These columns can define the observability by inspecting the position of the zero inputs. If all the $i$-th entries from all orthonormal set of basis vectors are zero then adding any combination of the null space to particular solution do not change the solution of the $i$-th state variable (nodal voltage). For this reason, the nodal voltage is among observable busbars. Otherwise, since the nodal voltages corresponding to non-zero entries cannot be uniquely defined they are classified among unobservable busbars. Therefore, SVD provides OA as a by-product by means of inspecting the null space vectors before or during the transient state estimation.

\section{Voltage Sag/Dip origin identification:}

TSE can be used to determine the location or source of transients in a power system. This can be performed by inspection of nodal mismatch voltage within the observable part of the network. Fig. 3 shows this procedure. Since unexpected fault event is not in the network model, the different between estimated state variables and calculated state variable will lead to voltage mismatch which can be used to identify the fault/disturbance type and location.

Given the fact that TSE provides time domain estimation, it can be used to assess voltage dip/sag as well as identifying different cause of voltage sag by inspecting the estimated voltage waveform [26]. However, in this paper, it is assumed that the cause of voltage sag is fault current.

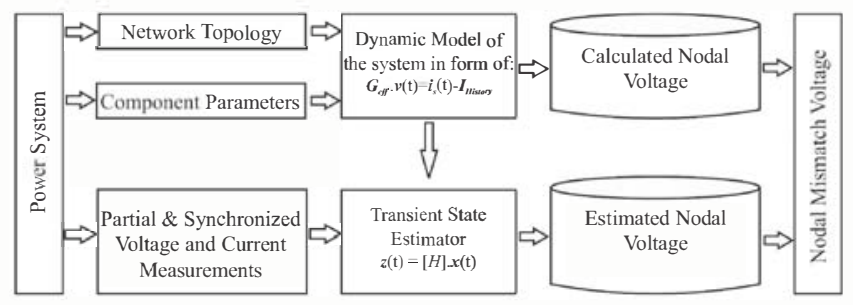

Fig. 3: Nodal mismatch voltage calculation

\section{CASE STUDY}

The test system is an $11 \mathrm{kV}$ distribution network taken from Killinchy area, a rural area in south Canterbury, in the South Island of New Zealand. The system consists of a ring of $11 \mathrm{kV}$ overhead lines and the lateral outgoing feeders. Symmetrical 
measurements are arbitrary placed at points indicated in the network (see Fig. 4). To consider the measurement noise, 5\% measurement noise (normally distributed at all measurement points) was also added to the obtained voltage and current measurements.

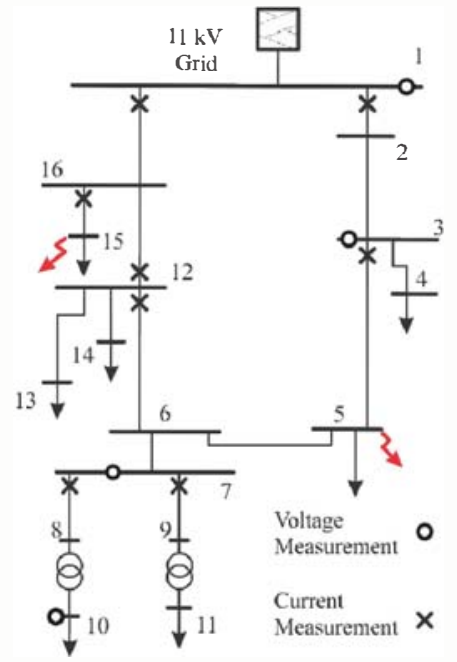

Fig. 4: Test system and measuring placement

The relevant test system components modelled in this case study are as follows:

a. Transmission lines are modelled by three-phase PI model with coupled elements.

b. Transformers are modelled by three ideal single-phase transformer represented by a mutual inductance coupling between windings. Connection matrices are used to derive the nodal equation based on coil configuration (e.g., Delta/Star, Star/Star, etc).

c. The real and reactive power components of the static loads are modelled by their equivalent resistance and inductance, respectively.

Due to the lack of field measurements a PSCAD/EMTDC simulation is used to generate the field data (hereafter called measured or actual values). The results from the simulation at the measurement points are then fed to the developed transient state estimator (TSE) and the estimated quantities at the unmonitored locations are compared to the simulation results for these locations. For this purpose, two single-phase short circuits at busbar No. 5 and busbar No.15 are simulated. This could simulate the case which is likely to happen in storm related events. There are no measurements on or near these busbars. A three-phase voltage measuring at busbar 10 displays an approximately $70 \%$ retained voltage on phases A $\&$ B. This is to investigate the accuracy of implemented TSE and its ability to identify the cause of the observed voltage dip/sag.

It should be noted that the number of measurements are 36 (nodes) compared to 48 unknown state variables (nodes). It results in an under-determined estimation problem, hence observable and unobservable busbars are predicted. Using SVD only gives reliable answers for observable nodes.

Fig. 5 illustrates a comparison between measured and estimated voltages at unmonitored nodes as a 3-D plot, to give an overview of the accuracy of the estimation of the whole system. It includes all nodes, both observable and unobservable. The unobservable nodes $(10,11 \& 12$ which are part of busbar 4 as a three-phase representation) are clearly evident by the error between the estimated and actual and the observability analysis (discussed in section II-B) also indicates these nodes are not observable.
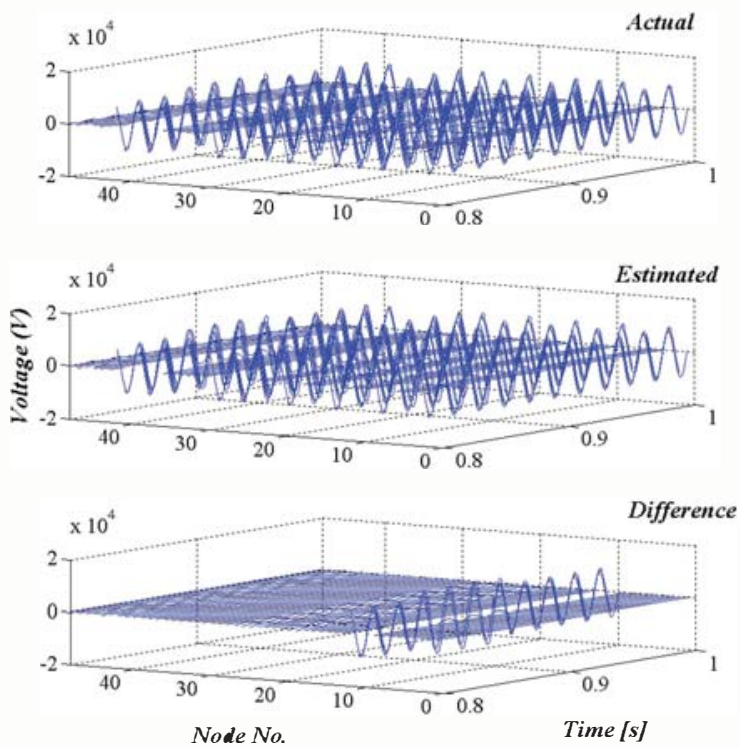

Fig. 5: Transient state estimation results.

The TSE simulation can be used to determine the location or source of transients in a power system. Fig. 6 shows maximum nodal mismatch voltage values for observable nodes during the study time. It depicts the maximum difference between the simulations of TSE, with and without the disturbance. It can be observed from Fig. 6 that the node with the largest change in voltage are the nodes 5 and 15, indicating that these nodes are most likely the source of the transient recorded at busbar 10 .

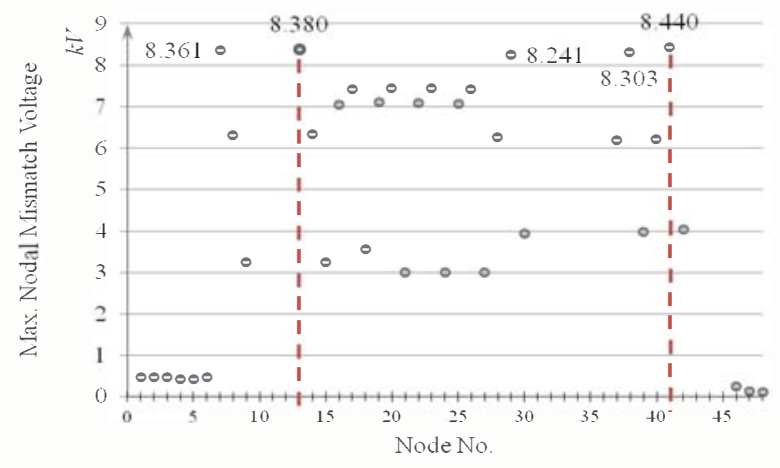

Fig. 6: Maximum nodal mismatch voltage values for observable nodes.

Fig. 7-10 shows the three-phase actual and estimated voltages for the suspicious location (busbars No. 5 and 15) as well as difference between actual and estimated voltages. The actual and estimated results are plotted as solid and dotted 
lines, respectively. However, they are indistinguishable due to the similarity. Inspecting the waveform also confirms that the voltage sag was occurred owing to single phase short circuits at phases $\mathrm{A}$ and $\mathrm{B}$ from busbars 5 and 15 , respectively.

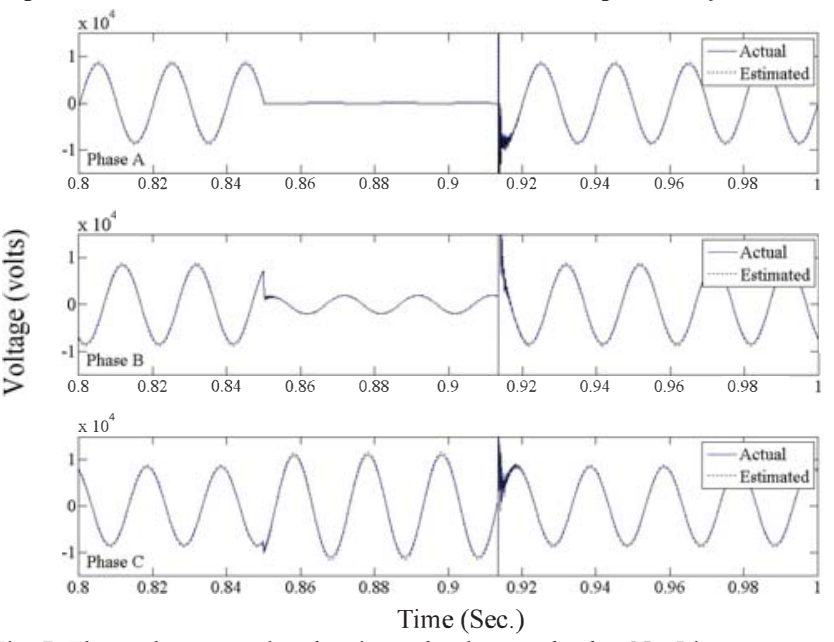

Fig. 7: Three-phase actual and estimated voltage at busbar No.5 in presence of $5 \%$ measurement noise.

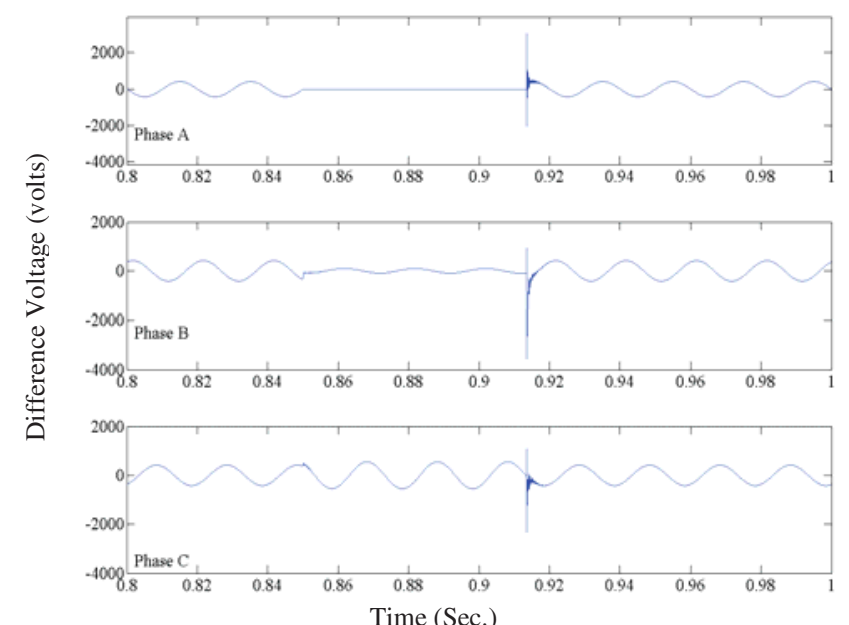

Fig. 8: Difference between actual and estimated voltages at busbar No. 5 in presence of $5 \%$ measurement noise

\section{CONCLUSION}

Due to the fact that TSE provides time domain estimation it could alternatively be used for many applications in power system in conjunction with signal processing techniques. This is more pronounced with the emerge of smart grids and associated Advanced Metering Infrastructure (AMI) which enables power systems access to a wealth of data that is becoming available. However, as Smart Grids evolves the number of measurements is not likely to be the barrier it once was due to the massive amount of data that is becoming available.

In this paper, the use of TSE for voltage sag/dip assessment using nodal mismatch voltage was discussed. The results clarified the accuracy of the method to identify the cause of transient occurred in a realistic distribution test system even in presence of measurement noise.
More research needs to be done for TSE becoming a commercial tool including refinements to the models further and test it more thoroughly. In particular incorporating travelling-wave transmission line model is important to increase the methods applicability to transmission systems.

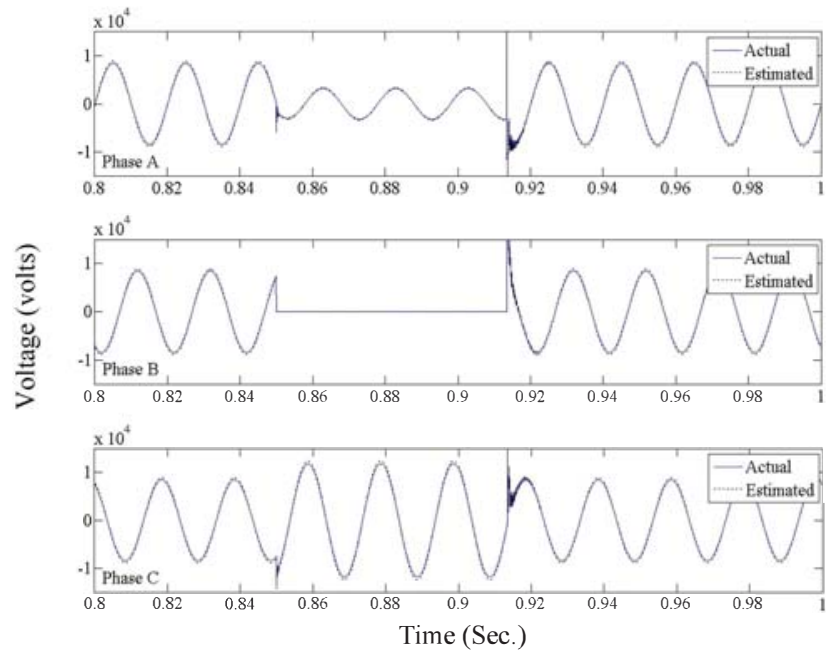

Fig. 9: Three-phase actual and estimated voltage at busbar No.15 in presence of $5 \%$ measurement noise.

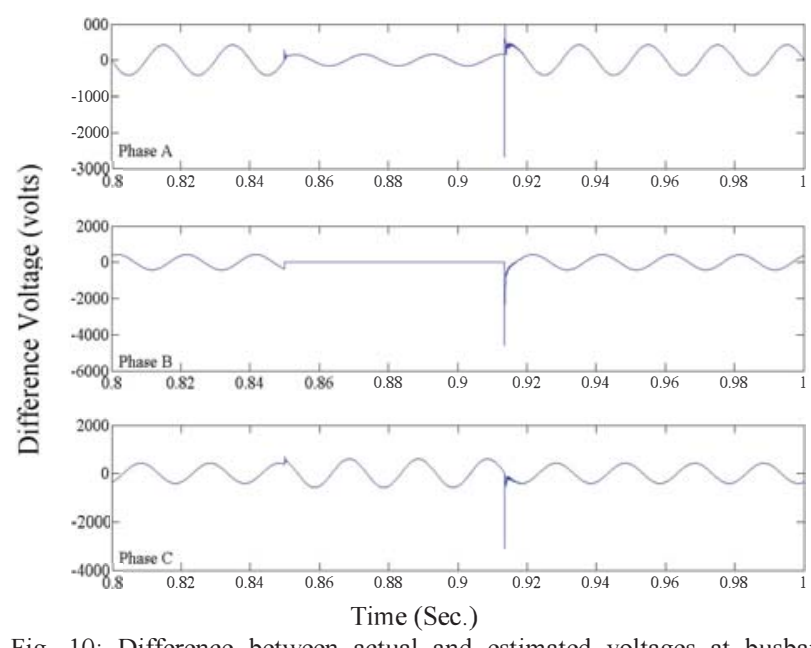

Fig. 10: Difference between actual and estimated voltages at busbar No. 15 in presence of $5 \%$ measurement noise.

\section{REFERENCES}

[1] "IEEE Recommended Practice for Monitoring Electric Power Quality," IEEE Std 1159-1995, p. i, 1995.

[2] L. Conrad, K. Little, and C. Grigg, "Predicting and preventing problems associated with remote faultclearing voltage dips," Industry Applications, IEEE Transactions on, vol. 27, pp. 167-172, 1991.

[3] M. H. J. Bollen, "Fast assessment methods for voltage sags in distribution systems," Industry Applications, IEEE Transactions on, vol. 32, pp. 1414-1423, 1996.

[4] L. E. Conrad and M. H. J. Bollen, "Voltage sag coordination for reliable plant operation," Industry Applications, IEEE Transactions on, vol. 33, pp. 14591464, 1997. 
[5] M. H. J. Bollen, "Method of critical distances for stochastic assessment of voltage sags," Generation, Transmission and Distribution, IEE Proceedings-, vol. 145, pp. 70-76, 1998.

[6] M. R. Qader, M. H. J. Bollen, and R. N. Allan, "Stochastic prediction of voltage sags in a large transmission system," Industry Applications, IEEE Transactions on, vol. 35, pp. 152-162, 1999.

[7] S. O. Faried and S. Aboreshaid, "Stochastic evaluation of voltage sags in series capacitor compensated radial distribution systems," Power Delivery, IEEE Transactions on, vol. 18, pp. 744-750, 2003.

[8] P. Heine and M. Lehtonen, "Voltage sag distributions caused by power system faults," Power Systems, IEEE Transactions on, vol. 18, pp. 1367-1373, 2003.

[9] S. O. Faried, R. Billinton, and S. Aboreshaid, "Stochastic evaluation of voltage sag and unbalance in transmission systems," Power Delivery, IEEE Transactions on, vol. 20, pp. 2631-2637, 2005.

[10] J. Wang, S. Chen, and T. T. Lie, "System voltage sag performance estimation," Power Delivery, IEEE Transactions on, vol. 20, pp. 1738-1747, 2005.

[11] D. G. Hart, et al., "Tapping protective relays for power quality information," Computer Applications in Power, IEEE, vol. 13, pp. 45-49, 2000.

[12] A. C. Parsons, W. M. Grady, E. J. Powers, and J. C. Soward, "A direction finder for power quality disturbances based upon disturbance power and energy," Power Delivery, IEEE Transactions on, vol. 15, pp. 1081-1086, 2000.

[13] C. Li, T. Tayjasanant, W. Xu, and X. Liu, "Method for voltage-sag-source detection by investigating slope of the system trajectory," Generation, Transmission and Distribution, IEE Proceedings-, vol. 150, pp. 367-372, 2003.

[14] N. Hamzah, A. Mohamed, and A. Hussain, "A new approach to locate the voltage sag source using real current component," Electric Power Systems Research, vol. 72, pp. 113-123, 2004.

[15] A. Seon-Ju, W. Dong-Jun, D. Y. Chung, and U. M. Seung, "Determination of the relative location of voltage sag source according to event cause," in Power Engineering Society General Meeting, 2004. IEEE, 2004, pp. 620-625 Vol.1.

[16] J. Gomez, M. Morcos, D. Tourn, and M. Felici, "A novel methodology to locate originating points of voltage sags in electric power systems," in Electricity Distribution, 2005. CIRED 2005. 18th International Conference and Exhibition on, 2005, pp. 1-3.

[17] A. K. Pradhan and A. Routray, "Applying distance relay for voltage sag source detection," Power Delivery, IEEE Transactions on, vol. 20, pp. 529-531, 2005.

[18] T. Tayjasanant, L. Chun, and W. Xu, "A resistance signbased method for voltage sag source detection," Power Delivery, IEEE Transactions on, vol. 20, pp. 2544-2551, 2005.
[19] R. C. Leborgne, D. Karlsson, and J. Daalder, "Voltage Sag Source Location Methods Performance Under Symmetrical and Asymmetrical Fault Conditions," in Transmission \& Distribution Conference and Exposition: Latin America, 2006. TDC '06. IEEE/PES, 2006, pp. 1-6.

[20] N. R. Watson, "Power quality state estimation," European Transactions on Electrical Power, vol. 20, pp. 19-33, Jan 2010.

[21] K. K. C. Yu and N. R. Watson, "An approximate method for transient state estimation," Ieee Transactions on Power Delivery, vol. 22, pp. 1680-1687, Jul 2007.

[22] N. R. Watson and K. K. C. Yu, "Transient State Estimation," in 13th International Conference on Harmonics and Quality of Power(ICHQP 2008), 2008, pp. 1-6.

[23] H. W. Dommel, "Digital Computer Solution of Electromagnetic Transients in Single- and Multiphase Networks," Ieee Transactions on Power Apparatus and Systems, vol. Pa88, pp. 388-\&, 1969.

[24] H. W. Dommel, Electromagnetic Transients Program Reference Manual (EMTP Theory Book). Portland: Bonneville Power Administration, 1986.

[25] W. H. Press, Numerical recipes : the art of scientific computing, 3rd ed. Cambridge, UK ; New York: Cambridge University Press, 2007.

[26] M. H. J. Bollen, I. Y. H. Gu, P. G. V. Axelberg, and E. Styvaktakis, "Classification of underlying causes of power quality disturbances: deterministic versus statistical methods," EURASIP J. Appl. Signal Process., vol. 2007, pp. 172-172, 2007.

\section{BIOGRAPHIES}

Ali Farzanehrafat was born in Tehran, Iran where he received B.Sc. and M.Sc. in power engineering in 2004 and 2007, respectively. He worked in Industries for 6 years. Currently, he is a visiting research student at University of Wollongong, Australia, while pursuing $\mathrm{PhD}$ degree at Electrical and Computer Engineering Department, University of Canterbury, Christchurch, New Zealand.

His area of research is Power Quality State Estimation.

Neville R. Watson (SM'99) gain his B.E. (Hons) and Ph.D. degrees in Electrical \& Electronic Engineering from the University of Canterbury in 1984 \& 1988 respectively, where he is now a Professor.

His main research area is computer simulation and analysis applied to power quality issues. In particular, harmonics, voltage fluctuations and electromagnetic transients. He has a special interest in modelling nonlinear loads and their interactions, from CFLs to HVDC schemes and aluminium smelters. More recently VSDs have been the focus of his work.

S. Perera (M'95) received the B.Sc.(Eng.) degree in electrical power engineering from the University of Moratuwa, Sri Lanka, the M.Eng.Sc degree in electrical engineering from the University of New South Wales, Australia, in 1978, and the Ph.D. degree in electrical engineering from the University of Wollongong, Wollongong, Australia, in 1988. He has been a Lecturer at the University of Moratuwa, Sri Lanka. Currently, he is an Associate Professor with the University of Wollongong. He is the Technical Director of the Endeavour Energy Power Quality and Reliability Centre, University of Wollongong. 